\title{
Abnormal glucose tolerance post-gestational diabetes mellitus as defined by the International Association of Diabetes and Pregnancy Study Groups criteria
}

\author{
Eoin Noctor ${ }^{1}$, Catherine Crowe ${ }^{1}$, Louise A Carmody', Jean A Saunders ${ }^{2}$, \\ Breda Kirwan', Angela O'Dea ${ }^{3}$, Paddy Gillespie, Liam G Glynn³, Brian E McGuire5, \\ Ciarán O'Neill' ${ }^{4}$, P M O'Shea ${ }^{6}$ and F P Dunne ${ }^{1}$ for the ATLANTIC-DIP investigators \\ ${ }^{1}$ Galway Diabetes Research Centre, National University of Ireland, Galway, Ireland, ${ }^{2}$ Statistical Consulting \\ Unit/CSTAR @ UL, University of Limerick, Limerick, Ireland, ${ }^{3}$ Department of General Practice, ${ }^{4}$ School of Business \\ and Economics, ${ }^{5}$ School of Psychology, National University of Ireland, Galway, Ireland, and ${ }^{6}$ Department of Clinical \\ Biochemistry, University Hospital Galway, Galway, Ireland
}

\author{
Correspondence \\ should be addressed \\ to E Noctor \\ Email \\ eoin.noctor@hse.ie
}

\begin{abstract}
Objective: An increase in gestational diabetes mellitus (GDM) prevalence has been demonstrated across many countries with adoption of the International Association of the Diabetes and Pregnancy Study Groups (IADPSG) diagnostic criteria. Here, we determine the cumulative incidence of abnormal glucose tolerance among women with previous GDM, and identify clinical risk factors predicting this.

Design: Two hundred and seventy women with previous IADPSG-defined GDM were prospectively followed up for 5 years (mean 2.6) post-index pregnancy, and compared with 388 women with normal glucose tolerance (NGT) in pregnancy. Methods: Cumulative incidence of abnormal glucose tolerance (using American Diabetes Association criteria for impaired fasting glucose, impaired glucose tolerance and diabetes) was determined using the Kaplan-Meier method of survival analysis. Cox regression models were constructed to test for factors predicting abnormal glucose tolerance. Results: Twenty-six percent of women with previous GDM had abnormal glucose tolerance vs $4 \%$ with NGT, with the log-rank test demonstrating significantly different survival curves $(P<0.001)$. Women meeting IADPSG, but not the World Health Organization (WHO) 1999 criteria, had a lower cumulative incidence than women meeting both sets of criteria, both in the early post-partum period $(4.2 \%$ vs $21.7 \%, P<0.001)$ and at longer-term follow-up $(13.7 \%$ vs $32.6 \%, P<0.001)$. Predictive factors were glucose levels on the pregnancy oral glucose tolerance test, family history of diabetes, gestational week at testing, and BMI at follow-up.

Conclusions: The proportion of women developing abnormal glucose tolerance remains high among those with IADPSG-defined GDM. This demonstrates the need for continued close follow-up, although the optimal frequency and method needs further study.

European Journal of Endocrinology

(2016) 175, 287-297
\end{abstract}

\section{Introduction}

The first diagnostic criteria for gestational diabetes mellitus (GDM) were published by O'Sullivan and Mahan in 1964 (1). These criteria (and their modified forms: the National Diabetes Data Group (2) and CarpenterCoustan criteria) (3) identified women at greatly increased risk of future diabetes (cumulative incidence
() 2016 European Society of Endocrinology Printed in Great Britain
Published by Bioscientifica Ltd. 
$60 \%$ at 16 years) (4). The World Health Organization (WHO) criteria (1980, revised 1985 and 1999) (5) used the same criteria as for diagnosis of diabetes mellitus and impaired glucose tolerance (IGT) in non-pregnant adults. Therefore, none was based on the prediction of pregnancy outcomes. In 2010, the International Association of Diabetes and Pregnancy Study Groups (IADPSG) proposed new diagnostic criteria (6) based on perinatal outcomes, using data from the Hyperglycemia and Adverse Pregnancy Outcomes (HAPO) study (7). However, given the paucity of data on the effect of application of the new criteria on clinical outcomes, as balanced against the resource and clinical implications of the increase in GDM prevalence seen with the newer criteria $(17.8 \%$, range $9.8-25.5 \%$, across HAPO study sites) (8), debate regarding their adoption continues. While WHO have adopted these criteria (9), the National Institutes of Health (10) and American College of Obstetricians and Gynecologists (11) have both recommended to retain the Carpenter-Coustan criteria, while the American Diabetes Association (ADA) adopted the IADPSG criteria in 2011 (12), and now present them as an alternative to the Carpenter-Coustan/National Diabetes Data Group criteria (13). Given the on-going debate, any data on foetal or maternal outcomes, both perinatally and longer-term, when the new criteria are applied, will be useful. However, as cohorts defined using the new criteria include women with milder degrees of glycaemic abnormality than those identified using older criteria, the longer-term risk of abnormal glucose tolerance may be less than when the older criteria are used.

Using IADPSG criteria, our group has shown a $12.4 \%$ prevalence of GDM, employing universal screening, as compared with $9.4 \%$ using the WHO 1999 criteria (14), while $19 \%$ of women with IADPSG-defined GDM demonstrated abnormal glucose tolerance (defined as impaired fasting glucose (IFG), IGT, or type 2 diabetes, using ADA criteria) at testing up to 6 months after delivery (15). Our primary objective in this study was to determine the cumulative incidence of abnormal glucose tolerance up to 5 years post-partum (the time period during which the cumulative incidence of diabetes shows the most marked increase) (16) in a cohort of women meeting IADPSG criteria for GDM. Our secondary aim was to identify which routinely available clinical variables are useful in predicting which women are more likely to progress to abnormal glucose tolerance.

\section{Subjects and methods}

From January 2007 to December 2010, women attending five antenatal care centres were screened for GDM using a one-step method (75 g oral glucose tolerance test (OGTT) with fasting, 1 and 2-h values). Women who met IADPSG criteria for the diagnosis of GDM from four of the five centres were eligible for inclusion in this study (ATLANTIC-DIP 2: GDM screening and intervention in the community). The remaining centre was not included due to practical and resource constraints. Women were invited to participate using an invitation letter, with a follow-up telephone call if there was no response. If a participant failed to attend a scheduled appointment, they were contacted again by telephone, and invited to re-attend.

The original study period (during the index pregnancy) for this cohort included an 18-month period of universal screening (all pregnant women presenting for antenatal care with an estimated last menstrual period between September 2007 and March 2009 were offered a 2-h $75 \mathrm{~g}$ OGTT). Selective screening (women with GDM risk factors only) was otherwise used. Women underwent an OGTT before the 24- to 28-week window if there was a previous history of GDM or if glycosuria was present, with a second OGTT performed at 24-28 weeks if the first was negative. Modified WHO 1999 diagnostic criteria for IFG/GDM (fasting plasma glucose $(\mathrm{FPG}) \geq 6.1 \mathrm{mmol} / \mathrm{L}, 2$-h glucose $\geq 7.8 \mathrm{mmol} / \mathrm{L}$ ) were in clinical use during the study. If GDM was diagnosed, women received dietary and lifestyle advice and were instructed in self-monitoring of blood glucose (SMBG). If fasting $(<5.3 \mathrm{mmol} / \mathrm{L})$ and 1 -h postprandial $(<7.8 \mathrm{mmol} / \mathrm{L})$ glucose targets were not met, insulin was commenced. Although modified WHO criteria were in clinical use at the time of the index pregnancy, women were classified retrospectively as either GDM or normal glucose tolerance (NGT) according to the IADPSG criteria (fasting $\geq 5.1 \mathrm{mmol} / \mathrm{L}, 1-\mathrm{h} \geq 10.0 \mathrm{mmol} / \mathrm{L}, 2$ - $\mathrm{h}$ $\geq 8.5 \mathrm{mmol} / \mathrm{L}$ ).

Exclusion criteria were current pregnancy and nonwhite European ethnicity. The rationale for the latter exclusion criterion was to control for the potential bias of ethnicity; although women of non-white ethnicity accounted for only $7.1 \%$ of our population screened for GDM, they accounted for $16.7 \%$ of GDM diagnoses. Inclusion of this group would be likely therefore to overestimate risk for the majority of our population. Also, as our focus was on the progression to IFG, IGT 
and type 2 diabetes among women with previous GDM, we excluded women with a clinical diagnosis of type 1 diabetes following the index pregnancy ( $n=3$ in the GDM group), who are likely to have a different underlying pathophysiology. A randomly selected control group, tested during the same period, but not meeting IADPSG criteria, and using the same exclusion criteria, was also invited (NGT group). Of note, women with twin pregnancies were included in the analysis.

All women with GDM were invited back for a $75 \mathrm{~g}$ OGTT at 12 weeks post-partum, as part of routine care, and were discharged back to their general practitioner if diabetes was not diagnosed. Women known to have type 2 diabetes, impaired glucose tolerance, or IFG $(n=42)$ at this post-partum test were scheduled for retesting for the purposes of the current study with FPG and haemoglobin A1c (HbA1c) only rather than a $75 \mathrm{~g}$ OGTT and HbA1c. These women were included in the analysis as meeting the primary outcome for this study. Following an 8- to 12-h fast, and informed consent, participants underwent a $75 \mathrm{~g}$ OGTT, with plasma glucose values taken at 0 and $120 \mathrm{~min}$. Samples for lipid profile, haemoglobin A1c (HbA1c) and Homeostatic Model Assessment 2Insulin Resistance (HOMA2-IR) determination were also obtained, detailed results of which have been reported in separate papers $(17,18)$.

The ADA criteria (12) for diabetes (fasting glucose $\geq 7.0 \mathrm{mmol} / \mathrm{L}$ or 2 -h glucose $\geq 11.1 \mathrm{mmol} / \mathrm{L}$ ), IFG (fasting glucose $5.6-6.9 \mathrm{mmol} / \mathrm{L})$ and IGT (2-h glucose 7.8 $11.0 \mathrm{mmol} / \mathrm{L}$ ) were used to diagnose abnormal glucose tolerance, in line with Irish national clinical guidelines (19). A family history of diabetes was defined as having either a first- or second-degree relative with diabetes. Blood pressure was the mean of two readings (Philips M3 physiological monitor), obtained 5 min apart while sitting quietly. Weight and height were recorded (Seca 635 scales), and waist circumference was measured (WHO technique). Laboratory samples for plasma glucose were analysed using the hexokinase method (Roche Modular P Analytics Chemistry Systems). The between-run coefficient of variation for glucose at mean concentrations of $2.56,7.11$ and $16.7 \mathrm{mmol} / \mathrm{L}$ were $1.1,0.9$ and $1.0 \%$ respectively. HbA1c was measured using the Menarini HA8160 automated analyser, employing reverse-phase cation exchange chromatography, and calibrated in accordance with the International Federation for Clinical Chemistry standards. Total and HDL-cholesterol, and serum triglycerides, were determined using the Roche Modular Analytics $<$ P $>$ Chemistry Systems, while LDLcholesterol was calculated using the Friedewald equation.

\section{Ethics committee approval}

Relevant regional research ethics committee approval (Clinical Research Ethics Committee, Galway University Hospitals) was obtained before recruitment.

\section{Statistical analysis}

Statistical analysis was carried out using SPSS version 20 and Minitab version 15 (State College, PA, USA). Cumulative incidence of abnormal glucose tolerance was determined using the Kaplan-Meier method of survival analysis to account for time elapsed between delivery and retesting for abnormal glucose tolerance. To investigate if women progressing to abnormal glucose tolerance post-partum could be identified on the basis of clinical risk factors assessed at the time of delivery of the index pregnancy, we constructed Cox proportional hazards regression models, using forward stepwise entry with a pre-selected set of predictive factors, with abnormal glucose tolerance up to 5 years post-partum as the outcome variable. We constructed two main explanatory models: one using variables available at the time of delivery only and one including variables available at follow-up testing for this study (Supplementary Material and Supplementary Table 1, see section on supplementary data given at the end of this article). We also constructed models including and excluding women who had abnormal glucose tolerance at the 12-week post-partum test, in order to control for the effect of time since delivery, as women with persistent abnormalities on the OGTT at 12 weeks post-partum are likely to have a greater degree of abnormal glucose tolerance than those who are normal initially, but develop abnormal glucose tolerance later. Only variables contributing significantly to the fit were included in the final models. Student's $t$-test and Mann-Whitney $U$ test were used to assess between-group differences in means and medians respectively of baseline characteristics. Statistical significance was set at $P<0.05$.

\section{Results}

We identified 4405 potential participants (491 meeting IADPSG criteria for GDM, 3914 with NGT) from the 5500 universally screened participants in the original ATLANTIC-DIP study (Fig. 1). In addition, we identified 874 (184 with IADPSG-defined GDM, 690 with NGT) women who were tested using selective screening. A total of 675 women of white European origin were identified 


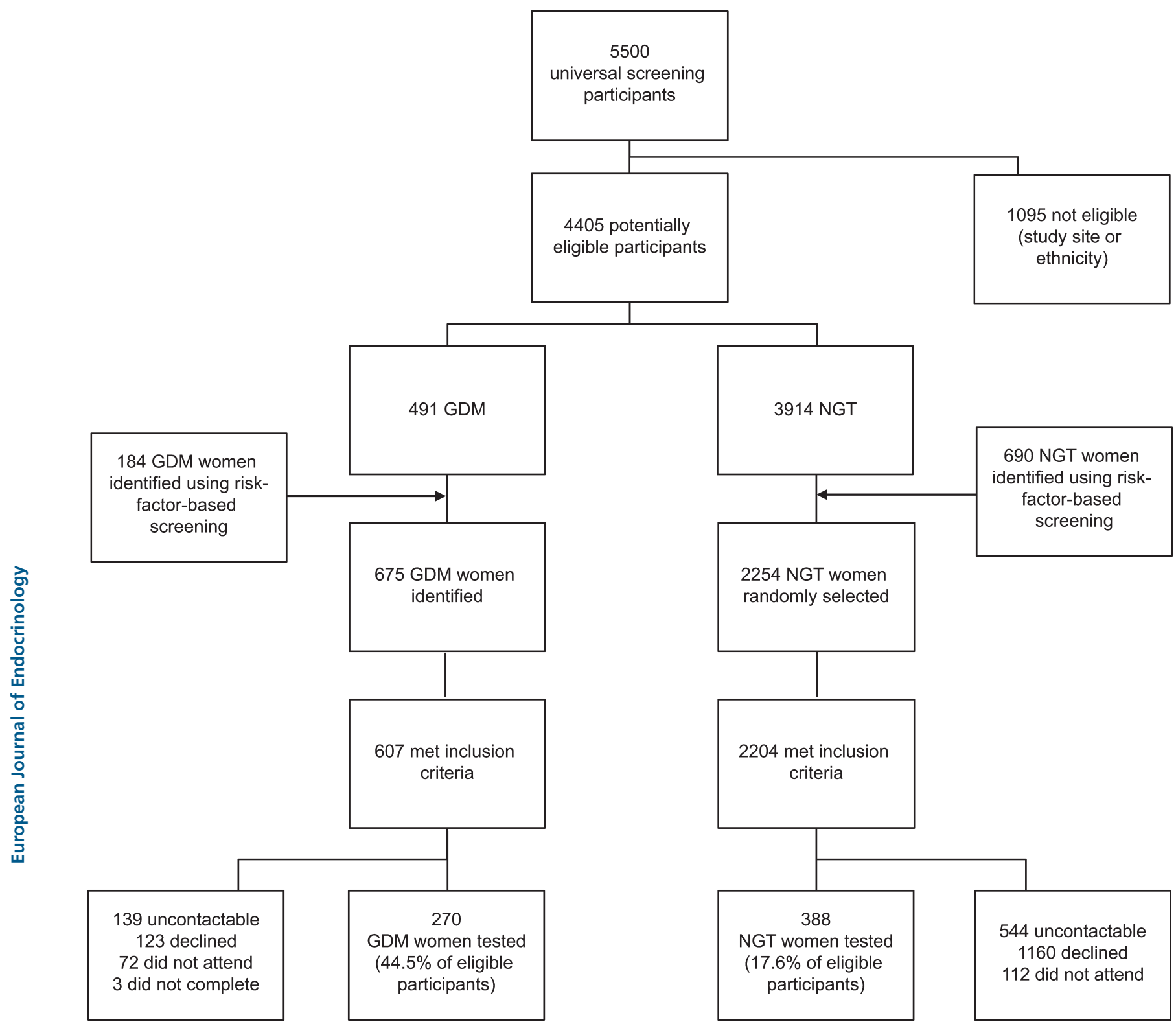

Figure 1

Flow diagram for study participants. GDM, gestational diabetes mellitus; NGT, normal glucose tolerance.

who met IADPSG GDM criteria between 2007 and 2010. Of these women, 607 met the inclusion criteria for this study, while 262 declined or could not be contacted. Of those agreeing to participate, 72 (21\%) did not attend, while 3 did not complete testing. Two hundred seventy women ( $45 \%$ of eligible population) attended and completed retesting. Of the control group of 2254 women invited, 500 agreed to participate - 112 (22\%) did not attend, while 388 completed retesting.

Comparison of the groups at retesting for this study is shown in Table 1. The mean duration of follow-up was shorter in women with previous IADPSG-defined GDM (2.6 vs 3.3 years in women with NGT, $P<0.001$ ). Women with IADPSG-defined GDM were younger than those with NGT (36.6 vs 37.6 years, $P=0.022$ ). Nine percent of women with IADPSG-defined GDM had undergone the index pregnancy OGTT before 24 weeks gestation, as compared with $5 \%$ with IADPSG-defined NGT $(P=0.046)$. Women with previous GDM had a higher BMI, higher glucose and HbA1c values; a more adverse lipid profile; and higher blood pressure. They also had higher mean glucose values at each point on the pregnancy OGTT, 
Table 1 Characteristics of participants meeting criteria for IADPSG-defined GDM and NGT. Figures represent means (s.D.) unless otherwise stated.

\begin{tabular}{l} 
Variable \\
\hline Characteristics at the time of the index pregnancy \\
Age in years at time of delivery in index pregnancy \\
BMI in $\mathrm{kg} / \mathrm{m}^{2}$ at time of pregnancy OGTT* \\
SBP in $\mathrm{mmH}$ at time of pregnancy OGTT** \\
DBP in mmHg at time of pregnancy OGTT** \\
Gestational week OGTT performed \\
Pregnancy fasting glucose (mmol/L) \\
1-h glucose (mmol/L) \\
2-h glucose (mmol/L) \\
Proportion using insulin in index pregnancy \\
Offspring birth weight in kg \\
Pre-eclampsia or pregnancy-induced hypertension in \\
index pregnancy (\%) \\
Polyhydramnios (\%) \\
Congenital malformation present (\%) \\
Shoulder dystocia (\%) \\
Neonatal death (\%) \\
Neonatal intensive care unit admission required (\%) \\
Caesarean section (\%) \\
Emergency Caesarean section (\%) \\
Breastfeeding status relating to index pregnancy \\
Any breastfeeding (\%) \\
Continued post hospital discharge (\%) \\
Continued 1 month or more (\%) \\
Continued 3 months or more (\%) \\
Characteristics at retesting for this study \\
Age (years) \\
Years since delivery \\
BMI (kg/m²) \\
Waist circumference (cm) \\
Fasting glucose (mmol/L) \\
2-h glucose (mmol/L) \\
HbA1c IFCC (mmol/mol) \\
HbA1c DCCT (\%) \\
Total cholesterol (mmol/L) \\
Triglycerides (mmol/L) \\
HDL-cholesterol (mmol/L) \\
LDL-cholesterol (mmol/L) \\
SBP (mmHg) \\
DBP (mmHg) \\
Proportion with family history of diabetes (\%) \\
\end{tabular}

\begin{tabular}{|c|c|}
\hline GDM $(n=270)$ & NGT $(n=388)$ \\
\hline $34.0(5.0)$ & $34.2(5.1)$ \\
\hline $31.3(6.6)$ & $27.1(4.3)$ \\
\hline 122.8 (12.3) & $119.1(12.2)$ \\
\hline $73.9(8.7)$ & $71.0(8.8)$ \\
\hline $27.3(4.2)$ & $26.4(2.6)$ \\
\hline $5.16(0.89)$ & $4.31(0.32)$ \\
\hline 10.37 (1.73) & 6.75 (1.61) \\
\hline $8.13(2.06)$ & $5.42(1.29)$ \\
\hline $35.6 \%$ & $2.1 \%$ \\
\hline $3.47(0.69)$ & $3.56(0.62)$ \\
\hline $24.9 \%$ & $11.0 \%$ \\
\hline $7.8 \%$ & $2.8 \%$ \\
\hline $1.5 \%$ & $4.1 \%$ \\
\hline $0.7 \%$ & $3.1 \%$ \\
\hline $0.4 \%$ & $1.8 \%$ \\
\hline $22.2 \%$ & $14.9 \%$ \\
\hline $47.8 \%$ & $26.8 \%$ \\
\hline $19.3 \%$ & $11.3 \%$ \\
\hline $59 \%$ & $55.2 \%$ \\
\hline $45.6 \%$ & $53.4 \%$ \\
\hline $41.5 \%$ & $44.6 \%$ \\
\hline $31.9 \%$ & $35.8 \%$ \\
\hline $36.6(5.0)$ & $37.6(5.1)$ \\
\hline $2.6(1.0)$ & $3.3(0.7)$ \\
\hline $29.7(6.9)$ & $26.1(4.9)$ \\
\hline $93.2(16.3)$ & $84.5(12.5)$ \\
\hline $5.07(0.95)$ & $4.65(0.41)$ \\
\hline $5.62(1.75)$ & $4.85(1.02)$ \\
\hline $36.2(4.8)$ & $33.6(2.7)$ \\
\hline $5.56(0.45)$ & $5.32(0.26)$ \\
\hline $5.0(1.0)$ & $4.7(0.8)$ \\
\hline $1.3(0.9)$ & $0.9(0.5)$ \\
\hline $1.5(0.4)$ & $1.6(0.4)$ \\
\hline $2.9(0.8)$ & $2.7(1.0)$ \\
\hline 124.7 (15.4) & $116.1(13.4)$ \\
\hline 73.6 (10.4) & $68.8(9.2)$ \\
\hline $65.2 \%$ & $50.0 \%$ \\
\hline
\end{tabular}

95\% Cl for difference $\overline{P \text { value for difference }}$$$
\text { -1.0. } 0.6
$$$$
\text { 3.2, } 5.1
$$$$
1.7,5.8
$$$$
1.5,4.4
$$$$
0.4,1.5
$$$$
0.74,0.96
$$$$
3.4,3.9
$$$$
2.4,3.0
$$$$
27.7,39.5
$$$$
-0.20,0.01
$$$$
\text { 8.1, } 20.1
$$$$
1.5,9.0
$$$$
-0.1,5.3
$$$$
0.0,4.7
$$$$
-0.5,3.0
$$$$
1.3,13.5
$$$$
13.5,28.2
$$$$
2.4,13.8
$$$$
-3.8,11.5
$$$$
0.0,15.4
$$$$
-4.5,10.7
$$$$
-3.4,11.2
$$

0.626

$<0.001$

$<0.001$

$<0.001$

0.001

$<0.001$

$<0.001$

$<0.001$

$<0.001$

0.076

$<0.001$

$0.13,1.70$

$0.6,0.9$

$<0.001$

$2.6,4.5$

$6.4,11.1$

$<0.001$

$0.30,0.54$

$0.53,1.02$

$<0.001$

$<0.001$

$<0.001$

$1.9,3.2$

$<0.001$

$0.18,0.30$

$<0.001$

$0.2,0.5$

$<0.001$

$0.3,0.5$

$0.1,0.2$

$0.1,0.4$

$<0.001$

$<0.001$

$<0.001$

$6.4,11.0$

$<0.001$

$3.2,6.3$

$7.5,22.5$

$1<0.001$ $<0.001$

*Incomplete data set $-10 \%$ of GDM and $16 \%$ of NGT participants did not have values recorded; **Incomplete data set - 15\% of GDM and $16 \%$ of NGT participants did not have values recorded; ${ }^{5}$ Note some women with WHO-defined GDM are defined as NGT using the IADPSG criteria, and were prescribed insulin during the index pregnancy.

DBP, diastolic blood pressure; GDM, gestational diabetes mellitus; HbA1c, haemoglobin A1c; IADPSG, International Association of Diabetes in Pregnancy Study Groups; NGT, normal glucose tolerance; OGTT, oral glucose tolerance test; SBP, systolic blood pressure.

and were more likely to have a family history of diabetes. There was no significant difference in the number of multiple pregnancies between the GDM and NGT groups (3\% vs $2.1 \%, P=0.526)$.

As compared with women with IADPSG-defined GDM participating in this study, women with IADPSG-defined GDM who participated in the original ATLANTIC-DIP study, but did not attend for the current study $(n=318)$, were of similar age, had a similar BMI during pregnancy, and similar fasting glucose values (Supplementary Table 2). They did, however, have significantly lower 1 and 2-h glucose values on the pregnancy OGTT. Women with IADPSG-defined NGT attending this study $(n=388)$, compared with those who did not, and had full data available from the index pregnancy $(n=3563)$, were significantly older, had a significantly higher BMI during 
pregnancy, had significantly higher 2-h glucose levels on the pregnancy OGTT, and were significantly more likely to have a family history of diabetes (Supplementary Table 3).

\section{Cumulative incidence of abnormal glucose tolerance}

The survival curves in Fig. 2 demonstrate the cumulative probability of abnormal glucose tolerance at follow-up for this study, split by the IADPSG criteria into women meeting NGT and GDM criteria. When the GDM and NGT groups were tested for differences using the logrank test, their survival curves were significantly different $(P<0.001)$. The differences remained significant when adjusted for age, BMI and family history of diabetes $(P<0.001)$. Of 270 women with previous GDM by IADPSG criteria, $10.4 \%$ met the criteria for a new diagnosis of abnormal glucose tolerance (Table 2). In addition to the $15.6 \%(n=42)$ of women already known to have abnormal glucose tolerance at initial post-partum testing, this yielded a cumulative incidence of abnormal glucose tolerance of $25.9 \%(n=70)$ in women with prior IADPSGdefined GDM, compared with 3.6\% $(n=14)$ of those with NGT $(P=0.001)$. Of the 270 women with prior GDM, at the time abnormal glucose tolerance was diagnosed, $12.2 \%(n=33)$ had IFG, 5.9\% $(n=16)$ had IGT, 5.6\% $(n=15)$ had combined IFG/IGT, while $2.2 \%(n=6)$ had diabetes. Of 388 women with previous NGT, $1.8 \%(n=7)$ had IFG, $1.5 \%(n=6)$ had IGT, $0.3 \%$ had combined IFG/IGT $(n=1)$, and none had diabetes.

\section{Prediction of progression to abnormal glucose tolerance}

The Cox proportional hazards regression results are summarised in Table 3 . Within the models including pregnancy variables only, fasting glucose and 1-h glucose values on the pregnancy OGTT, and family history of diabetes, proved the strongest predictors on both models. Of these, when all participants were included, family history had the highest hazard ratio at $2.04(P=0.014)$. Both fasting glucose and 1-h glucose values on the pregnancy OGTT were highly significant. With regard to timing of testing during the pregnancy, earlier gestation at diagnosis of GDM became significant only when women diagnosed at the 12-week post-partum visit were included. Family history of diabetes, fasting glucose and 1-h glucose all remained significantly associated with abnormal glucose tolerance when participants with abnormal glucose tolerance at 12 weeks were removed.
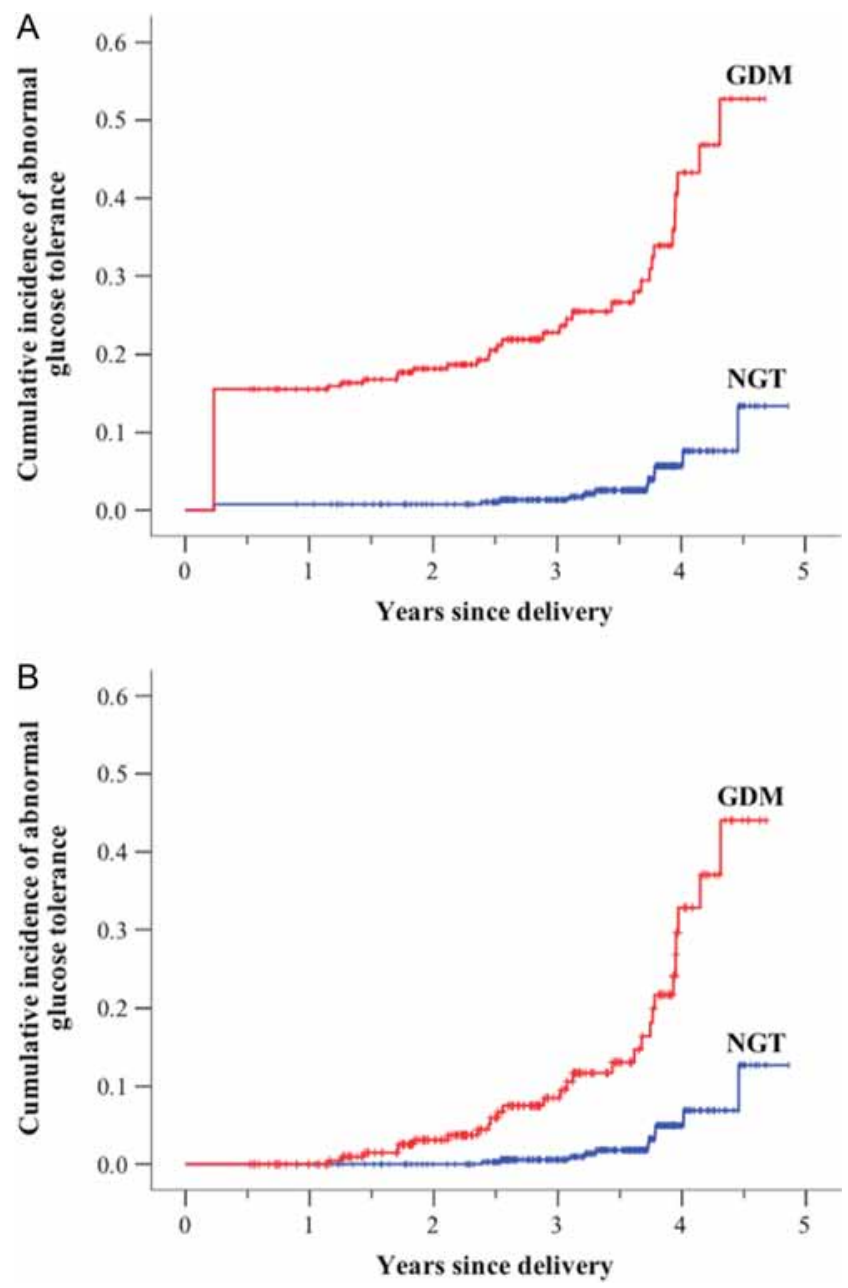

Figure 2

Kaplan-Meier curves showing the cumulative probability of abnormal glucose tolerance in women with previous IADPSG-defined GDM vs those with IADPSG-defined NGT. (A) includes all participants (GDM $n=270, n=388$ ), while (B) excludes women known to have abnormal glucose tolerance at 12 week post-partum testing (GDM $n=228$, NGT $n=385$ ). Abnormal glucose tolerance is defined as any one of impaired fasting glucose, impaired glucose tolerance or diabetes, using the American Diabetes Association criteria. GDM, gestational diabetes mellitus; IADPSG, International Association of the Diabetes and Pregnancy Study Groups; NGT, normal glucose tolerance.

The model including all participants showed a loglikelihood ratio (-2LL) of 673.726 (4df, $P<0.001$ ), while the model excluding participants with abnormal glucose tolerance at 12 weeks had a $-2 \mathrm{LL}$ of 238.984 , 3df, $P<0.001$; the lower value indicating a better overall fit. When we constructed models including variables available at 
Table 2 Proportion of women with abnormal glucose tolerance at 12-week post-partum testing and at retesting for the current study.

\begin{tabular}{|c|c|c|c|c|}
\hline & GDM $(n=270)$ & NGT $(n=388)$ & 95\% Cl for difference & $P$ value for difference \\
\hline \multicolumn{5}{|c|}{ Number of participants with abnormal glucose tolerance at early post-partum testing } \\
\hline IFG & 18 & 0 & & \\
\hline IGT & 8 & 2 & & \\
\hline IFG/IGT & 12 & 1 & & \\
\hline DM & 4 & 0 & & \\
\hline Total early post-partum & $42(15.6 \%)$ & $3(0.8 \%)$ & $10.7,19.6$ & 0.001 \\
\hline \multicolumn{5}{|c|}{ Number of participants with newly diagnosed abnormal glucose tolerance at this study } \\
\hline IFG & 15 & 7 & & \\
\hline IGT & 8 & 4 & & \\
\hline IFG/IGT & 3 & 0 & & \\
\hline DM & 2 & 0 & & \\
\hline Total for this study & $28(10.4 \%)$ & $11(2.8 \%)$ & $3.8,11.9$ & $<0.001$ \\
\hline Total number & $70(25.9 \%)^{\dagger}$ & $14(3.6 \%)$ & $16.9,28.0$ & 0.001 \\
\hline
\end{tabular}

${ }^{\dagger}$ Added and calculated percentages differ slightly due to rounding error.

DM, diabetes mellitus; GDM, gestational diabetes mellitus; IFG, impaired fasting glucose; IGT, impaired glucose tolerance; NGT, normal glucose tolerance.

follow-up for this study, again, index pregnancy OGTT values (fasting, 1- and 2-h) and family history of diabetes were significant predictors, while BMI at follow-up was the only variable available since the index pregnancy associated with development of abnormal glucose tolerance. Twohour glucose was significant only when women with abnormal glucose at 12 weeks post-partum were included in the model. With regard to models including variables available at follow-up, the model including all participants showed a -2LL of 806.84 (5df, $P<0.001$ ), while the model including women at follow-up only showed a better overall fit, with a -2LL of 338.07 (4df, $P<0.001$ ).

\section{Comparison of modified WHO 1999 vs IADPSG criteria}

When data on women attending for this study were analysed according to the modified WHO 1999 criteria in clinical use at the time of the study, 214 women met modified WHO 1999 criteria for GDM diagnosis, while 444 met NGT criteria (Supplementary Table 4 for figures for IADPSG and modified WHO criteria). The cumulative incidence of abnormal glucose tolerance was $29.4 \%$ among women with WHO-defined GDM and $4.7 \%$ among women with WHO-defined NGT. Neither of these proportions differed significantly from those seen when

Table 3 Predictive factors for the presence of abnormal glucose tolerance at any time up to 5 years post-index pregnancy ( $n=658$ ) and abnormal glucose tolerance developing after the 12 -week post-partum test $(n=613)$. Two separate Cox proportional hazards regression models were constructed for each of these; the first using variables available at the time of delivery only, and the second using variables from the first model (BMI and age replaced with BMI and age at follow-up), with variables available post-delivery included.

\begin{tabular}{|c|c|c|c|c|}
\hline \multirow[b]{3}{*}{ Variable } & \multicolumn{2}{|c|}{ AGT up to 5 years $(n=658)$} & \multicolumn{2}{|c|}{ AGT at later follow-up only $(n=613)$} \\
\hline & $\begin{array}{l}\text { Index pregnancy } \\
\text { variables only }\end{array}$ & $\begin{array}{l}\text { Including variables } \\
\text { at follow-up }\end{array}$ & $\begin{array}{l}\text { Index pregnancy } \\
\text { variables only }\end{array}$ & $\begin{array}{l}\text { Including variables } \\
\text { at follow-up }\end{array}$ \\
\hline & $\mathrm{HR}(95 \% \mathrm{Cl})$ & $\mathrm{HR}(95 \% \mathrm{Cl})$ & $\mathrm{HR}(95 \% \mathrm{Cl})$ & $\mathrm{HR}(95 \% \mathrm{Cl})$ \\
\hline $\begin{array}{l}\text { Fasting glucose on } \\
\text { pregnancy OGTT }\end{array}$ & $1.69(1.29,2.21) P<0.001$ & $1.52(1.13,2.06) P=0.006$ & $2.01(1.27,3.20) P=0.003$ & $1.99(1.27,3.12) P=0.003$ \\
\hline $\begin{array}{l}\text { 1-h glucose on } \\
\text { pregnancy OGTT }\end{array}$ & $1.23(1.07,1.41) P=0.004$ & $1.27(1.11,1.45) P=0.001$ & $1.25(1.02,1.54) P=0.032$ & $1.31(1.09,1.57) P=0.004$ \\
\hline $\begin{array}{l}\text { 2-h glucose on } \\
\text { pregnancy OGTT }\end{array}$ & $1.1(0.99,1.23) P=0.082$ & $1.15(1.02,1.29) P=0.021$ & $1.07(0.90,1.28) P=0.444$ & $1.08(0.91,1.77) P=0.397$ \\
\hline $\begin{array}{l}\text { Week of gestation at } \\
\text { pregnancy OGTT }\end{array}$ & $0.94(0.90,1.00) P=0.032$ & $0.95(0.90,1.07) P=0.068$ & $0.94(0.88,1.01) P=0.092$ & $0.95(0.88,1.01) P=0.103$ \\
\hline Family history & $2.04(1.16,3.62) P=0.014$ & $1.81(1.04,3.15) P=0.035$ & $2.94(1.17,7.41) P=0.022$ & $2.45(1.07,5.59) P=0.033$ \\
\hline BMI at follow-up & $N / A$ & $1.04(1.01,1.29) P=0.009$ & N/A & $1.05(1.00,1.09) P=0.036$ \\
\hline
\end{tabular}

HR represents increase per $1 \mathrm{mmol} / \mathrm{L}(18 \mathrm{mg} / \mathrm{dL})$ of glucose concentration; per week of gestation, and per $1 \mathrm{~kg} / \mathrm{m}^{2}$ increase in BMI. AGT, abnormal glucose tolerance; GDM, gestational diabetes mellitus; HR, hazard ratio; OGTT, oral glucose tolerance test. 
IADPSG criteria were used $(P=0.390$ for women with GDM, $P=0.421$ for women with NGT). One hundred and seventy-five women met both IADPSG and modified WHO 1999 criteria, 95 met IADPSG criteria only, 39 met modified WHO 1999 criteria only, and 349 met neither IADPSG nor modified WHO criteria. Women meeting both IADPSG and modified WHO 1999 criteria had a significantly greater cumulative incidence of abnormal glucose tolerance at follow-up than those meeting IADPSG criteria alone $(32.6 \%$ vs $13.7 \%, P<0.001)$, or women meeting modified WHO 1999 criteria alone (32.6\% vs $15.4 \%, P=0.033)$. There was no significant difference seen in cumulative incidence between women meeting IADPSG criteria only and those meeting modified WHO 1999 criteria only $(P=0.798)$. Comparison of baseline characteristics for women meeting IADPSG criteria only and those meeting both IADPSG and modified WHO criteria are also shown in Supplementary Table 5.

\section{Risk factor-based vs universal screening}

Of note, our cohort contains a group of women identified by risk factor-based screening. However, only $9 \%(n=18)$ of women with IADPSG-defined GDM in this cohort did not have at least one risk factor for GDM, of whom only three developed abnormal glucose tolerance by the time of this study. Overall, no difference was seen in the proportion of women with abnormal glucose tolerance post-delivery (24.6\% identified during risk factor-based screening vs $26.3 \%$, for those identified during universal screening, $P=0.782$ ), although it should be noted that due to the study design, time since delivery was shorter in the risk factor-screened group (1.3 vs 3.0 years, $P<0.01$ ). Women identified as having IADPSG-defined GDM during the universal screening period, when compared with those identified using risk factor-based screening, were slightly younger at delivery (33.6 (s.D. 5.0) vs 35.2 (s.D. 4.8) years, $P=0.024)$, and had a lower (10.12 (s.D. 1.74$)$ vs 11.2 (s.D. $1.42) \mathrm{mmol} / \mathrm{L}, P<0.001) 1-\mathrm{h}$, but similar fasting (5.15 (s.D. $0.85)$ vs 5.18 (s.D. 1.02$) \mathrm{mmol} / \mathrm{L}, P=0.790)$ and 2 -h $(8.04$ (s.D. 2.02) vs 8.39 (s.D. 2.19) $\mathrm{mmol} / \mathrm{L}, P=0.235$ ) glucose values (Supplementary Table 6).

\section{Discussion}

Our results show that, despite the IADPSG criteria being based on adverse pregnancy outcome, they still define a cohort at significantly increased future risk of abnormal glucose tolerance. Over a quarter demonstrate abnormal glucose tolerance at this relatively short postpartum interval, as compared with women with normal glucose tolerance in pregnancy. Of note, the women with normal glucose tolerance are representative of the Irish background population in this age group, showing a similar prevalence of abnormal glucose tolerance (20). As expected, given less strict diagnostic thresholds, those women who meet the IADPSG criteria alone are at lower (although still relatively high; prevalence of $14 \%$ ) risk of abnormal glucose tolerance at a mean of 2.6 years postindex pregnancy, as compared with women attending for this study who also meet the older modified WHO criteria. Of note also, 2-h glucose on the pregnancy OGTT demonstrates a less strong relationship than the fasting or 1 -h value. This may be of clinical relevance; despite the increased threshold of 2-h glucose for diagnosis of GDM, as compared with the modified 1999 WHO criteria, the risk of abnormal glucose tolerance at follow-up remains similar. It should also be noted that women meeting modified WHO criteria (but not women meeting IADPSG criteria alone) would have received information about the future risk of diabetes at the time of the post-partum visit. Although no structured post-partum lifestyle programme was in place, we cannot exclude that the general advice given by either the hospital team, or the patient's primary care physician, regarding weight management after delivery could have influenced progression to abnormal glucose tolerance in this cohort. Further prospective data on women meeting IADPSG criteria only will be useful in clarifying this issue.

The factors associated with progression to abnormal glucose tolerance are similar to those noted in previous studies using older criteria - pregnancy OGTT values (fasting glucose in particular) $(16,21,22,23,24)$ and elevated BMI after pregnancy $(25,26)$. Earlier diagnosis of GDM, noted in previous studies as a risk factor (22, $24,27,28,29,30$ ), is associated with abnormal glucose tolerance at the first post-partum visit, but not with later progression. Family history of diabetes is associated with abnormal glucose tolerance, using both index pregnancy variables, and variables available at follow-up, and whether or not women with abnormal glucose tolerance at the 12-week test are included in the analysis. Family history has been identified as a risk factor (15, $31)$, although less consistently than the above factors. All of these factors are routinely available clinically, but cannot reliably discriminate between those women who require more frequent follow-up from those who do not. This is a clinically important point - identification of glucose abnormalities before progression to type 2 
diabetes is important in these women for two reasons. First, the Diabetes Prevention Program (32) showed a $71 \%$ increased crude incidence rate for women with a history of GDM versus those with no GDM history (38.4\% vs $25.7 \% 3$ years post-randomisation), despite similar characteristics at baseline, but also showed that treatment (lifestyle measures or metformin) reduced the rate of progression to type 2 diabetes (32). Second, detection of abnormal glucose tolerance in women considering further pregnancy allows timely intervention to attempt to reduce the risk of undiagnosed type 2 diabetes and the potential foetal complications associated with this presenting for the first time in a subsequent pregnancy (6).

A limitation of this study is the relatively low uptake on rescreening (45\%). However, comparing those women with IADPSG-defined GDM who did not attend for follow-up for this study with those who did (Supplementary Table 2), mean age at delivery, BMI during pregnancy and parity are similar. In contrast, 1and 2-h pregnancy OGTT levels are slightly higher, and insulin use during pregnancy was more prevalent in the group who attended for follow-up, potentially causing a selection bias toward the more severe GDM cases, which could in turn lead to overestimation of abnormal glucose tolerance at follow-up. There is, however, no difference in fasting glucose, which shows a stronger association with future abnormal glucose tolerance in both this and previous studies. Women with IADPSG-defined NGT attending for follow-up, as compared with those who did not, were older, more likely to have a family history of diabetes (Supplementary Table 3). These factors mean that that the prevalence of abnormal glucose tolerance in the NGT group may also overestimate the true prevalence of abnormal glucose tolerance. Low uptake on follow-up testing is a common issue among women with previous GDM, both for early (33) and long-term follow-up (34), and is a potential source of bias. To address this issue of low uptake on post-partum screening, we have initiated close follow-up of women with previous GDM with the help of a central coordinator for the region who makes verbal and written contact with each woman diagnosed with GDM to arrange early post-partum testing with a $75 \mathrm{~g}$ OGTT, and has achieved follow-up rates in excess of $75 \%$ (35). Further research into strategies for increasing longerterm follow-up rates will be valuable in determining the optimal approach to this issue. Also, our cohort contains women identified using risk factor-based screening, raising the possibility of bias due to the possibility of a more adverse risk profile, and therefore possibly a higher risk of glycaemic abnormalities at retesting. However, no difference in the cumulative incidence of abnormal glucose tolerance is seen here between the groups, and indeed, $91 \%$ of women screened during the universal screening period had at least one risk factor for GDM, which would have mandated screening if selective screening was used. It should be noted, however, that, due to study design, time since delivery was shorter in the risk factor-based screening group, raising the possibility that the cumulative incidence in this group may be longer if followed for as long as the universally screened group.

Finally, we did not include women of non-white European origin, leading to a potential bias. Women of non-white European ethnicity have previously been found to have a higher risk of progression to diabetes post-GDM, despite similar attendance for follow-up screening (36). Our group has previously shown that, of a group of 300 women with IADPSG-defined GDM screened at 3 months post-partum, $31 \%$ of women of non-white European ethnicity had abnormal glucose tolerance, versus only $18 \%$ of women of white European origin (15). Therefore, exclusion of these women is likely to underestimate the true risk of abnormal glucose tolerance in our population with IADPSG-defined GDM.

In summary, although it has been proposed that women meeting IADPSG criteria are likely to require less intensive follow-up for progression to type 2 diabetes than those meeting older criteria (37), our figures show that the risk remains high. Given that differentiation of those at lower risk cannot be reliably performed clinically, follow-up should be as frequent as for those women meeting the older criteria. Given the increase in numbers of GDM pregnancies associated with adoption of the IADPSG criteria, further study is essential to both determine the optimal method and frequency of follow-up.

\section{Supplementary data}

This is linked to the online version of the paper at http://dx.doi.org/10.1530/ EJE-15-1260.

\section{Declaration of interest}

The authors declare that there is no conflict of interest that could be perceived as prejudicing the impartiality of the research reported.

\section{Funding}

E N reports receiving an educational grant from Novo Nordisk Ireland Ltd. Funding for the study was provided by the Health Research Board (HRB) of Ireland (grant number ICE/2011/3). 


\section{Author contribution statement}

E N was involved in study design, collected and analysed data, and wrote the manuscript. C C was involved in study design, collected data and revised the manuscript for important intellectual content. L A C, B K, A O D, P G, $L G G, B E M, C O N$ and $P M O S$ were involved in study design and revised the manuscript for important intellectual content. J A $S$ conducted the statistical analysis, wrote the statistical methods section, and revised the manuscript for important intellectual content. F P D conceived the study, revised the manuscript for important intellectual content, had full access to the data and made the decision to publish.

\section{Acknowledgements}

ATLANTIC-DIP Collaborators: Dr G Gaffney, Ms B Wickham, University Hospital Galway; Dr M Durkan, Portiuncula Hospital Galway; Dr M Mohammed, Mayo General Hospital; Dr N Ravikumar; Ms T Gallacher, Letterkenny General Hospital.

\section{References}

1 O'Sullivan JB \& Mahan CM. Criteria for the oral glucose tolerance test in pregnancy. Diabetes 196413 278-285.

2 National Diabetes Data Group. Classification and diagnosis of diabetes mellitus and other categories of glucose intolerance. Diabetes 197928 1039-1057. (doi:10.2337/diab.28.12.1039)

3 Carpenter MW \& Coustan DR. Criteria for screening tests for gestational diabetes. American Journal of Obstetrics and Gynecology 1982144 768-773. (doi:10.1016/0002-9378(82)90349-0)

4 O'Sullivan JB. Establishing criteria for gestational diabetes. Diabetes Care 19803 437-439. (doi:10.2337/diacare.3.3.437)

5 World Health Organisation. Definition and Diagnosis of Diabetes Mellitus and Intermediate Hyperglycaemia. Geneva, Switzerland: World Health Organisation, 2006.

6 Metzger BE, Gabbe SG, Persson B, Buchanan TA, Catalano PA, Damm P, Dyer AR, Leiva A, Hod M, Kitzmiler JL et al. International association of diabetes and pregnancy study groups recommendations on the diagnosis and classification of hyperglycemia in pregnancy. Diabetes Care 201033 676-682. (doi:10.2337/dc09-1848)

7 Metzger BE, Lowe LP, Dyer AR, Trimble ER, Chaovarindr U, Coustan DR, Hadden DR, McCance DR, Hod M, McIntyre HD et al. Hyperglycemia and adverse pregnancy outcomes. New England Journal of Medicine 2008358 1991-2002. (doi:10.1056/nejmoa0707943)

8 Sacks DA, Hadden DR, Maresh M, Deerochanawong C, Dyer AR, Metzger BE, Lowe LP, Coustan DR, Hod M, Oats JJ et al. Frequency of gestational diabetes mellitus at collaborating centers based on IADPSG consensus panel-recommended criteria: the Hyperglycemia and Adverse Pregnancy Outcome (HAPO) Study. Diabetes Care 2012 35 526-528. (doi:10.2337/dc11-1641)

9 World Health Organization. Diagnostic Criteria and Classification of Hyperglycaemia First Detected in Pregnancy. Geneva, Switzerland: World Health Organization, 2013.

10 Noctor E, Crowe C, Carmody LA, Avalos GM, Kirwan B, Infanti JJ, O'Dea A, Gillespie P, Newell J, McGuire B et al. ATLANTIC DIP: simplifying the follow-up of women with previous gestational diabetes. European Journal of Endocrinology of the European Federation of Endocrine Societies 2013169 681-687. (doi:10.1530/EJE-13-0491)

11 Committee on Practice Bulletins-Obstetrics. Practice bulletin no. 137: gestational diabetes mellitus. Obstetrics and Gynecology 2013 122 406-416. (doi:10.1097/01.aog.0000433006.09219.f1)

12 American Diabetes Association. Standards of medical care in diabetes - 2011. Diabetes Care 201134 (Supplement 1) S11-S61. (doi:10.1016/0002-9378(82)90349-0)
13 American Diabetes Association. Standards of medical care in diabetes 2. Classification and diagnosis of diabetes. Diabetes Care 201639 (Supplement 1) S13-S22.

14 O'Sullivan EP, Avalos G, O'Reilly M, Dennedy MC, Gaffney G, Dunne F \& Atlantic DIPc. Atlantic Diabetes in Pregnancy (DIP): the prevalence and outcomes of gestational diabetes mellitus using new diagnostic criteria. Diabetologia 201154 1670-1675. (doi:10.1007/ s00125-011-2150-4)

15 O'Reilly MW, Avalos G, Dennedy MC, O'Sullivan EP \& Dunne F. Atlantic DIP: high prevalence of abnormal glucose tolerance post partum is reduced by breast-feeding in women with prior gestational diabetes mellitus. European Journal of Endocrinology 2011165 953-959. (doi:10.1530/EJE-11-0663)

16 Kim C, Newton KM \& Knopp RH. Gestational diabetes and the incidence of type 2 diabetes: a systematic review. Diabetes Care 2002 25 1862-1868. (doi:10.2337/diacare.25.10.1862)

17 Noctor E, Crowe C, Carmody LA, Avalos GM, Kirwan B, Infanti JJ, O'Dea A, Gillespie P, Newell J, McGuire B et al. ATLANTIC DIP: simplifying the follow-up of women with previous gestational diabetes. European Journal of Endocrinology 2013169 681-687. (doi:10.1530/EJE-13-0491)

18 Noctor E, Crowe C, Carmody LA, Kirwan B, O'Dea A, Glynn LG, McGuire BE, O'Shea PM \& Dunne FP. ATLANTIC-DIP: prevalence of metabolic syndrome and insulin resistance in women with previous gestational diabetes mellitus by International Association of Diabetes in Pregnancy Study Groups criteria. Acta Diabetologica 201552 153-160. (doi:10.1007/s00592-014-0621-z)

19 Harkins V. A Practical Guide to Integrated Type 2 Diabetes Care. Dublin: Health Service Executive, 2008.

20 Sinnott M, Kinsley BT, Jackson AD, Walsh C, O'Grady T, Nolan JJ, Gaffney P, Boran G, Kelleher C \& Carr B. Fasting plasma glucose as initial screening for diabetes and prediabetes in irish adults: the Diabetes Mellitus and Vascular health initiative (DMVhi). PLOS ONE 201510 e0122704. (doi:10.1371/journal.pone.0122704)

21 Buchanan TA, Xiang A, Kjos SL, Lee WP, Trigo E, Nader I, Bergner EA, Palmer JP \& Peters RK. Gestational diabetes: antepartum characteristics that predict postpartum glucose intolerance and type 2 diabetes in Latino women. Diabetes 199847 1302-1310. (doi:10.2337/diab.47.8.1302)

22 Catalano PM, Vargo KM, Bernstein IM \& Amini SB. Incidence and risk factors associated with abnormal postpartum glucose tolerance in women with gestational diabetes. American Journal of Obstetrics and Gynecology 1991165 914-919. (doi:10.1016/00029378(91)90438-W)

23 Metzger BE, Bybee DE \& Freinkel N. Gestational diabetes mellitus. Correlations between the phenotypic and genotypic characteristics of the mother and abnormal glucose tolerance during the first year postpartum. Diabetes 198534 111-115. (doi:10.2337/ diab.34.2.S111)

24 Kjos SL, Peters RK, Xiang A, Henry OA, Montoro M \& Buchanan TA. Predicting future diabetes in Latino women with gestational diabetes. Utility of early postpartum glucose tolerance testing. Diabetes $1995 \mathbf{4 4}$ 586-591. (doi:10.2337/diab.44.5.586)

25 Steinhart JR, Sugarman JR \& Connell FA. Gestational diabetes is a herald of NIDDM in Navajo women: high rate of abnormal glucose tolerance after GDM. Diabetes Care 199720 943-947. (doi:10.2337/ diacare.20.6.943)

26 Wang L, Liu H, Zhang S, Leng J, Liu G, Zhang C, Li WQ, Li N, Li W, $\mathrm{Li} \mathrm{Y}$ et al. Obesity index and the risk of diabetes among Chinese women with prior gestational diabetes. Diabetic Medicine 201431 1368-1377. (doi:10.1111/dme.12532)

27 Damm P, Kuhl C, Bertelsen A \& Molsted-Pedersen L. Predictive factors for the development of diabetes in women with previous gestational diabetes mellitus. American Journal of Obstetrics and Gynecology 1992 167 607-616. (doi:10.1016/S0002-9378(11)91559-2) 
28 Coustan DR, Carpenter MW, O'Sullivan PS \& Carr SR. Gestational diabetes: predictors of subsequent disordered glucose metabolism. American Journal of Obstetrics and Gynecology 1993168 1139-1144. (doi:10.1016/0002-9378(93)90358-p)

29 Lee AJ, Hiscock RJ, Wein P, Walker SP \& Permezel M. Gestational diabetes mellitus: clinical predictors and long-term risk of developing type 2 diabetes - a retrospective cohort study using survival analysis. Diabetes Care 200730 878-883. (doi:10.2337/dc06-1816)

30 Schaefer-Graf UM, Klavehn S, Hartmann R, Kleinwechter H, Demandt N, Sorger M, Kjos SL, Vetter K \& Abou-Dakn M. How do we reduce the number of cases of missed postpartum diabetes in women with recent gestational diabetes mellitus? Diabetes Care 200932 1960-1964. (doi:10.2337/dc09-0627)

31 Capula C, Chiefari E, Vero A, Foti DP, Brunetti A \& Vero R. Prevalence and predictors of postpartum glucose intolerance in Italian women with gestational diabetes mellitus. Diabetes Research and Clinical Practice 2014105 223-230. (doi:10.1016/j.diabres.2014.05.008)

32 Ratner RE, Christophi CA, Metzger BE, Dabelea D, Bennett PH, Pi-Sunyer X, Fowler S \& Kahn SE. Prevention of diabetes in women with a history of gestational diabetes: effects of metformin and lifestyle interventions. Journal of Clinical Endocrinology and Metabolism 200893 4774-4779. (doi:10.1210/jc.2008-0772)

33 Shah BR, Lipscombe LL, Feig DS \& Lowe JM. Missed opportunities for type 2 diabetes testing following gestational diabetes: a population-based cohort study. Obstetrical and Gynecological Survey 201267 137-139. (doi:10.1097/OGX.0b013e31824b6f2d)

34 Beischer NA, Wein P \& Sheedy MT. A follow-up program for women with previous gestational diabetes mellitus. Medical Journal of Australia 1997166 353-357.

35 Carmody L, Egan AM \& Dunne FP. Postpartum glucose testing for women with gestational diabetes mellitus: improving regional recall rates. Diabetes Research and Clinical Practice 2015108 e38-e41. (doi:10.1016/j.diabres.2015.04.005)

36 Girgis CM, Gunton JE \& Cheung NW. The influence of ethnicity on the development of type 2 diabetes mellitus in women with gestational diabetes: a prospective study and review of the literature. ISRN Endocrinology 20122012 341638. (doi:10.5402/2012/341638)

37 American Diabetes Association. Standards of medical care in diabetes - 2013. Diabetes Care 201336 (Supplement 1) S11-S66. (doi:10.2337/dc13-s011)

Received 28 December 2015

Revised version received 11 June 2016

Accepted 15 July 2016 